\title{
- Insights into the Developmental Journey of Robotic Medical Surgery
}

\section{Harish Kumar Banga}

Department of Mechanical Engineering, Guru Nanak Dev Engineering College, Ludhiana, Punjab India.

E-mail:drhkbanga@gmail.com

\section{INTRODUCTION}

Medical robotics has been in use for approximately 30 years. Robotic surgery is called "revolutionary" by many surgeons who value it for its many advantages, both real and potential. Its origin owes much to the weaknesses of the minimally invasive surgery (MIS) technologies that preceded it.

Robotic surgical operation yet clinical simulators hold dramatically modified the strategies because of the greater than having a whole bunch of common: each makes use of a mechanized interface as affords visible affected individual reactions among answer according to the strikes of the fitness care professional (although simulation additionally consists over touch feedback); every usage video show units after thinking out the development concerning the procedure; last, each utilizes personal computer software program reasons through which the health ponderable specialist interacts. Both utilized sciences are experiencing fast receiving or are regarded as like modalities that enable doctors to work in accordance with functioning more and extra tricky minimally invasive procedures even as enhancing affected individual safety.

Robotic surgeries are generally minimally invasive. This characteristic has been round prolonged previously than the beginning regarding robots. It is a widespread thought so encompasses dense standard procedures, such namely a laparoscopic cholecystectomy, and gall bladder excisions. The behavior refers to an approach so avoids lengthy cuts by means concerning deed concerning the physique through little (usually $1 \mathrm{~cm}$ ) entrance incisions. Surgeons use longhandled devices to function over the interior of the body. Such operations are guided by the usage of viewing equipment referred to in conformity with endoscopes. These are skinny tubes together with a DigiCam linked according to the cease on such so much approves the healthcare professional in conformity with considering highly magnified realtime 3-dimensional pictures about the process internet site on line over a monitor. The contemporary benefits over the robotic surgical process embody higher accuracy, precision, dexterity, tremor corrections, scaled motion, and larger these days haptic corrective feedback. These benefits stop end result into extra profitable surgical procedures and smaller indispensable intersection cuts. Overall, the robotic buildings bear higher truth or directness than unbacked surgeons. Surgical robots are successful in accordance with the role of the surgical gear nearer according to the "right spot" and deviate a lot less out of the "right trajectory." The robot's end-effectors do be a brush smaller than increased skills than an ethical hand. They perform file yet filter outdoors a surgeon's herbal limb briskness yet rescale motion after make bigger candidness yet reduce the chance because of error. Lastly, the robotic needs to keep in the surgeon's pace among undesired instructions via haptic feedback.

Researchers are formulating latter techniques in conformity with address job yet skill resistance. For example, the surgical robotic may additionally want in imitation of synchronically go together with a death coronary bravery such to that amount their supporter pace is suppressed according to zero. Another possible enhancement is the ability because of the robotic according to robotically Inure in conformity with the dynamical art arrest atop time so the sensitivity association on it tactics goes previous ethnic capabilities. Typically, the robotic surgical functions may be labeled as much each (i) supervisory-controlled, (ii) telesurgical, and (iii) shared-controlled. The supervisory-controlled method is near computerized concerning the ternary methods. The RoboDoc beside Integrated Surgical Systems Inc. is an occasion concerning a supervisory-controlled gadget ancient into orthopedic surgeries. After the overall merchant positions, the RoboDoc's bone-milling system at the right function inner the patient, the robotic mechanically cut the bone in conformity with virtually the honest measurement for the orthopedic implant.

Prior in accordance with the surgical procedure, the physician wants in imitation of put collectively the action through the planning yet registration phase. In the planning phase, photographs on the patient's frame are chronic in accordance with determining the proper surgical approach. Common imaging methods consist of laptop computer tomography (CT) 
scans, magnetic resonance imaging (MRI) scans, ultrasonography, fluoroscopy, and X-ray scans. Next, of the fixation phase, the fitness merchant ought in imitation of discovering the factors about the patient's corpse so much correspond in imitation of the photos constructed at the partial podium in the dodge phase. These factors are matched in imitation of a 3D model, who can be over to persimmon by way of using portraits considered by means of cameras or different realtime imaging strategies every via surgery. After the robotic find the amazing in the form of the mannequin yet reality, the surgical manner is performed.

The telesurgical strategy permits the surgical robotic to remain teleoperated, so is, operated out of a reach through an ethical surgeon. In practice, the robotic yet the healthcare expert are fully a doublet on meters apart. Tele-operation is additionally manageable all through big distances. However, issues certainly so period delays (i.e., telesurgical latencies) or the reachable bandwidth (i.e., the volume concerning facts so much perform lie transferred through soloist time) need after stand considered. The telesurgical method is ancient with the aid of course concerning the da Vinci Surgical System, which back according to stand invented by Philip S. Green then promoted with the aid of Intuitive Surgical Inc. This system in modern times dominates the surgical robotic market. Initially dubbed Mona (after Leonardo's Mona Lisa), the system used to be once rechristened the da Vinci Surgical Robot in 1999; among pursuance in conformity with Mr. Green "...in honor of the personality any had invented the advance robot." Although da Vinci via no skill invented then manufactured an authentic robotic (credit because of as goes after Tesla), he made many drawings on a variety of mechanisms

The da Vinci System consists of ternary principal components: (1) a viewing then manipulate explain so much is back including the useful resource of the surgeon, (2) a blazing yet prescient cart so much holds the endoscopes and offers seen remarks yet (3) a surgical robot's manipulator side unit so consists regarding ternary or four arms, relying on the model. The units to that amount are devoted according to the palms are especially specialized. Functions because of to them correspond over clamping, cutting, suturing, plantain manipulation, cauterizing, etc. It takes a partial period because surgeons after reach used to according to the da Vinci System. According to a study, too along with a preliminary teaching program, supplied through Intuitive Surgical, such takes as regards $12-18$ operations earlier than surgeons sense unembarrassed performing the procedure.

The shared-controlled method refers in conformity with the strategy via skill on which the robotic is no longer truly assignment teleoperated namely that execute determine out in accordance with standing up to the surgeons' meant pace postulate it deems up to the expectation it would in modern times now not be beneficial. Typically, the labor residence is smashed on between a range of segments and the system behaves within a one-of-a-kind pathway primarily based completely over certain on a sort localization of accordance in imitation of safe, close, boundary, and forbidden classification. For example, salvo physician strikes a reducing device of the route concerning plantain as want according to at last now not be damaged, the robotic intention acts the stress haptic feedback as will improve higher as like the reducing system comes nearer after the fragile tissue. In distinct words, here, scientific professional once more "feels" the digital instance over tissue up to expectation might also hold preprogrammed specs wonderful beyond the authentic adroitness namely properly as like rather one-of-a-kind localization in space.

\section{REFERENCES}

1. Gifari MW, Naghibi H, Stramigioli S, Abayazid M. A review on recent advances in soft surgical robots for endoscopic applications. Int J Med Robot Comp Assist Surg. 2019;15(5):e2010.

2. Ginoya T, Maddahi Y, Zareinia K. A historical review of medical robotic platforms. J Robot. 2021;2021:6640031. 